\title{
Positive Solutions for a Mixed-Order Three-Point Boundary Value Problem for $p$-Laplacian
}

\author{
Francisco J. Torres \\ Departamento de Matemática, Universidad de Atacama, Avenida Copayapu 485, Casilla 240, Copiapó, Región de Atacama, Chile \\ Correspondence should be addressed to Francisco J. Torres; ftorres@mat.uda.cl
}

Received 5 April 2013; Revised 30 July 2013; Accepted 3 October 2013

Academic Editor: Jaume Giné

Copyright (c) 2013 Francisco J. Torres. This is an open access article distributed under the Creative Commons Attribution License, which permits unrestricted use, distribution, and reproduction in any medium, provided the original work is properly cited.

The author investigates the existence and multiplicity of positive solutions for boundary value problem of fractional differential equation with $p$-Laplacian operator. The main tool is fixed point index theory and Leggett-Williams fixed point theorem.

\section{Introduction}

In this paper, we are interested in the existence of single and multiple positive solutions to nonlinear mixed-order threepoint boundary value problem for $p$-Laplacian. Consider the following:

$$
\begin{aligned}
& \left(\varphi_{p}\left(D_{0^{+}}^{\alpha} u(t)\right)\right)^{\prime}+a(t) f(t, u(t))=0, \quad 0<t<1, \\
& D_{0^{+}}^{\alpha} u(0)=u(0)=u^{\prime \prime}(0)=0, \quad u^{\prime}(1)=\gamma u^{\prime}(\eta)
\end{aligned}
$$

where $\eta, \gamma \in(0,1), \alpha \in(2,3], D_{0^{+}}^{\alpha}$ is the Caputo's derivative, $\varphi_{p}(s)=|s|^{p-2} s$, and $\varphi_{q}=\left(\varphi_{p}\right)^{-1}$ with $(1 / p)+(1 / q)=1$. We assume the following conditions throughout.

(H1) $f \in C([0,1] \times[0, \infty),[0, \infty))$.

(H2) $a \in L^{1}(0,1)$ is nonnegative and $a(t) \quad \not \equiv \quad 0$ on any subinterval of $(0,1)$.

The equation with a $p$-Laplacian operator arises in the modeling of different physical and natural phenomena, nonNewtonian mechanics, nonlinear elasticity and glaciology, combustion theory, population biology, nonlinear flow laws, and so on. Liang et al. in [1] used the fixed point theorem of Avery and Henderson to show the existence of at least two positive solutions. Zhao et al. [2] studied the existence of at least three positive solutions by using Leggett-Williams fixed point theorem. Chai [3] obtain results for the existence of at least one nonnegative solution and two positive solutions by using fixed point theorem on cone. Su et al. [4] studied the existence of one and two positive solutions by using the fixed point index theory. $\mathrm{Su}$ [5] studied the existence of one and two positive solution by using the method of defining operator by the reverse function of Green function and the fixed point index theory. Tang et al. [6] studied the existence of positive solutions of fractional differential equation with $p$-laplacian by using the coincidence degree theory.

Motivated by the above works, we obtain some sufficient conditions for the existence of at least one and three positive solutions for (1) and (2).

The organization of this paper is as follows. In Section 2, we present some necessary definitions and preliminary results that will be used to prove our results. In Section 3, we discuss the existence of at least one positive solution for (1) and (2). In Section 4, we discuss the existence of multiple positive solutions for (1) and (2). Finally, we give some examples to illustrate our results in Section 5.

\section{Preliminaries}

Definition 1. Let $E$ be a real Banach space. A nonempty closed convex set $K \subset E$ is called cone if

(1) $x \in K, \lambda \geq 0$ then $\lambda x \in K$,

(2) $x \in K,-x \in K$ then $x=0$.

Definition 2. An operator is called completely continuous if it is continuous and maps bounded sets into precompact sets. 
Remark 3. By the positive solution of (1) and (2) we understand a function $u(t)$ which is positive on $[0,1]$ and satisfies the differential equation (1) and the boundary conditions (2).

We will consider the Banach space $E=C[0,1]$ equipped with standard norm:

$$
\|u\|=\max _{0 \leq t \leq 1}|u(t)|
$$

The proof of existence of solution is based upon an application of the following theorem.

Theorem 4 (see $[7,8])$. Let $E$ be a Banach space and let $K$ be a cone of E. For $r>0$, define $K_{r}=\{u \in K:\|u\| \leq r\}$ and assume that $T: K_{r} \rightarrow K$ is a completely continuous operator such that $T u \neq u$ for all $u \in \partial K_{r}$.

(1) If $\|T u\| \leq\|u\|$ for all $u \in \partial K_{r}$, then $i\left(T, K_{r}, K\right)=1$.

(2) If $\|T u\| \geq\|u\|$ for all $u \in \partial K_{r}$, then $i\left(T, K_{r}, K\right)=0$.

Lemma 5 (see [9]). Let $n \in \mathbb{N}$ with $n \geq 2$, $n-1<\alpha \leq n$. If $u \in C^{n-1}[a, b]$ and $D_{a^{+}}^{\alpha} u \in C(a, b)$, then

$$
\left(I_{a^{+}}^{\alpha} D_{a^{+}}^{\alpha} u\right)(t)=u(t)-\sum_{k=0}^{n-1} \frac{u^{(k)}(a)}{k !}(t-a)^{k}
$$

holds on $(a, b)$.

Lemma 6. The three-point boundary value problem (1)-(2) has a unique solution

$$
\begin{aligned}
u(t)= & \int_{0}^{1} G_{1}(t, s) \varphi_{q}\left(\int_{0}^{s} a(\tau) f(\tau, u(\tau)) d \tau\right) d s \\
& +\frac{\gamma t}{1-\gamma} \int_{0}^{1} G_{2}(\eta, s) \\
& \times \varphi_{q}\left(\int_{0}^{s} a(\tau) f(\tau, u(\tau)) d \tau\right) d s,
\end{aligned}
$$

where

$$
\begin{aligned}
& G_{1}(t, s) \\
& \quad= \begin{cases}\frac{(\alpha-1) t(1-s)^{\alpha-2}-(t-s)^{\alpha-1}}{\Gamma(\alpha)}, & 0 \leq s \leq t \leq 1, \\
\frac{(\alpha-1) t(1-s)^{\alpha-2}}{\Gamma(\alpha)}, & 0 \leq t \leq s \leq 1,\end{cases} \\
& G_{2}(\eta, s) \\
& = \begin{cases}\frac{(\alpha-1)(1-s)^{\alpha-2}-(\alpha-1)(\eta-s)^{\alpha-2}}{\Gamma(\alpha)}, & 0 \leq s \leq \eta \leq 1, \\
\frac{(\alpha-1)(1-s)^{\alpha-2},}{\Gamma(\alpha)}, & 0 \leq \eta \leq s \leq 1 .\end{cases}
\end{aligned}
$$

Proof. Integrating both sides of (1) on $[0,1]$, we have

$$
\varphi_{p}\left(D^{\alpha} u(t)\right)-\varphi_{p}\left(D^{\alpha} u(0)\right)=-\int_{0}^{t} a(s) f(s, u) d s .
$$

So

$$
D^{\alpha} u(t)=-\varphi_{q}\left(\int_{0}^{t} a(s) f(s, u) d s\right) .
$$

From Lemma 5, we have

$$
\begin{aligned}
u(t)= & -\frac{1}{\Gamma(\alpha)} \int_{0}^{t}(t-s)^{\alpha-1} \varphi_{q}\left(\int_{0}^{s} a(\tau) f(\tau, u) d \tau\right) d s \\
& +A+B t+C t^{2}, \\
u^{\prime}(t)= & -\frac{1}{\Gamma(\alpha-1)} \int_{0}^{t}(t-s)^{\alpha-2} \varphi_{q}\left(\int_{0}^{s} a(\tau) f(\tau, u) d \tau\right) d s \\
& +B+2 C t, \quad \int^{\prime \prime}(t)=-\frac{1}{\Gamma(\alpha-2)} \int_{0}^{t}(t-s)^{\alpha-3} \varphi_{q} \\
& \times\left(\int_{0}^{s} a(\tau) f(\tau, u) d \tau\right) d s+2 C .
\end{aligned}
$$

From (2), $A=0$ and $C=0$.

Now, consider the following:

$$
\begin{array}{r}
u^{\prime}(1)=-\frac{1}{\Gamma(\alpha-1)} \int_{0}^{1}(1-s)^{\alpha-2} \varphi_{q} \\
\quad \times\left(\int_{0}^{s} a(\tau) f(\tau, u) d \tau\right) d s+B, \\
u^{\prime}(\eta)=-\frac{1}{\Gamma(\alpha-1)} \int_{0}^{\eta}(\eta-s)^{\alpha-2} \varphi_{q} \\
\quad \times\left(\int_{0}^{s} a(\tau) f(\tau, u) d \tau\right) d s+B,
\end{array}
$$

by the boundary value condition $u^{\prime}(1)=\gamma u^{\prime}(\eta)$, we have

$$
\begin{aligned}
B=\frac{1}{(1-\gamma) \Gamma(\alpha-1)} \int_{0}^{1}(1-s)^{\alpha-2} \varphi_{q} \\
\quad \times\left(\int_{0}^{s} a(\tau) f(\tau, u) d \tau\right) d s \\
-\frac{\gamma}{(1-\gamma) \Gamma(\alpha-1)} \int_{0}^{\eta}(\eta-s)^{\alpha-2} \varphi_{q} \\
\quad \times\left(\int_{0}^{s} a(\tau) f(\tau, u) d \tau\right) d s
\end{aligned}
$$

so

$$
\begin{aligned}
u(t)= & -\frac{1}{\Gamma(\alpha)} \int_{0}^{t}(t-s)^{\alpha-1} \varphi_{q}\left(\int_{0}^{s} a(\tau) f(\tau, u) d \tau\right) d s \\
& +\frac{t}{(1-\gamma) \Gamma(\alpha-1)}
\end{aligned}
$$




$$
\begin{aligned}
& \times \int_{0}^{1}(1-s)^{\alpha-2} \varphi_{q}\left(\int_{0}^{s} a(\tau) f(\tau, u) d \tau\right) d s \\
& -\frac{\gamma t}{(1-\gamma) \Gamma(\alpha-1)} \\
& \times \int_{0}^{\eta}(\eta-s)^{\alpha-2} \varphi_{q}\left(\int_{0}^{s} a(\tau) f(\tau, u) d \tau\right) d s .
\end{aligned}
$$

Splitting the second integral in two parts of the form

$$
\frac{t}{\Gamma(\alpha-1)}+\frac{k}{(1-\gamma) \Gamma(\alpha-1)}=\frac{t}{(1-\gamma) \Gamma(\alpha-1)},
$$

we have $k=\gamma t$; thus,

$$
\begin{aligned}
& u(t)=-\frac{1}{\Gamma(\alpha)} \int_{0}^{t}(t-s)^{\alpha-1} \varphi_{q} \\
& \quad \times\left(\int_{0}^{s} a(\tau) f(\tau, u) d \tau\right) d s \\
& +\frac{t}{\Gamma(\alpha-1)} \int_{0}^{1}(1-s)^{\alpha-2} \varphi_{q} \\
& \quad \times\left(\int_{0}^{s} a(\tau) f(\tau, u) d \tau\right) d s \\
& +\frac{\gamma t}{(1-\gamma) \Gamma(\alpha-1)} \int_{0}^{1}(1-s)^{\alpha-2} \varphi_{q} \\
& -\frac{\gamma t}{(1-\gamma) \Gamma(\alpha-1)} \int_{0}^{\eta}(\eta-s)^{\alpha-2} \varphi_{q} \\
& \times\left(\int_{0}^{s} a(\tau) f(\tau, u) d \tau\right) d s
\end{aligned}
$$

therefore,

$$
\begin{aligned}
u(t)= & \int_{0}^{t}\left(\frac{t(1-s)^{\alpha-2}}{\Gamma(\alpha-1)}-\frac{(t-s)^{\alpha-1}}{\Gamma(\alpha)}\right) \varphi_{q} \\
& \times\left(\int_{0}^{s} a(\tau) f(\tau, u) d \tau\right) d s \\
& +\int_{t}^{1} \frac{t(1-s)^{\alpha-2}}{\Gamma(\alpha-1)} \varphi_{q}\left(\int_{0}^{s} a(\tau) f(\tau, u) d \tau\right) d s \\
& +\frac{\gamma t}{1-\gamma} \int_{0}^{\eta}\left(\frac{(1-s)^{\alpha-2}}{\Gamma(\alpha-1)}-\frac{(\eta-s)^{\alpha-2}}{\Gamma(\alpha-1)}\right) \varphi_{q} \\
& +\frac{\gamma t}{1-\gamma} \int_{\eta}^{1} \frac{(1-s)^{\alpha-2}}{\Gamma(\alpha-1)} \varphi_{q}\left(\int_{0}^{s} a(\tau) f(\tau, u) d \tau\right) d s .
\end{aligned}
$$

This completes the proof.
Lemma 7. Let $\beta \in(0,1)$ be fixed. The kernel, $G_{1}(t, s)$, satisfies the following properties.

(1) $0 \leq G_{1}(t, s) \leq G_{1}(1, s)$ for all $s \in(0,1)$,

(2) $\min _{\beta \leq t \leq 1} G_{1}(t, s) \geq \beta G_{1}(1, s)$ for all $s \in[0,1]$.

Proof. (1) As $2<\alpha \leq 3$ and $0 \leq s \leq t \leq 1$, we have

$$
\begin{aligned}
& (\alpha-1) t(1-s)^{\alpha-2}>t(1-s)^{\alpha-2} \\
& \quad \geq(t-s)(t-s)^{\alpha-2}=(t-s)^{\alpha-1} ;
\end{aligned}
$$

thus, $G_{1}(t, s)>0$. Note $\partial G_{1}(t, s) / \partial t \geq 0$ then, $G_{1}(t, s)$ is increasing as a function of $t$; therefore,

$$
G_{1}(t, s) \leq G_{1}(1, s) \quad \forall s \in[0,1] .
$$

(2) For $\beta \leq t \leq 1$, we have

$$
\min _{\beta \leq t \leq 1} G_{1}(t, s)=G_{1}(\beta, s),
$$

where

$$
G_{1}(\beta, s)= \begin{cases}\frac{(\alpha-1) \beta(1-s)^{\alpha-2}-(\beta-s)^{\alpha-1}}{\Gamma(\alpha)} & 0 \leq s \leq \beta, \\ \frac{(\alpha-1) \beta(1-s)^{\alpha-2}}{\Gamma(\alpha)} & \beta \leq s \leq 1 .\end{cases}
$$

(a) If $0<s \leq \beta$,

$$
\min _{\beta \leq t \leq 1} G_{1}(t, s)=\frac{\beta(\alpha-1)(1-s)^{\alpha-2}}{\Gamma(\alpha)}-\frac{(\beta-s)^{\alpha-1}}{\Gamma(\alpha)} ;
$$

on the other hand,

$$
\beta G_{1}(1, s)=\frac{\beta(\alpha-1)(1-s)^{\alpha-2}}{\Gamma(\alpha)}-\frac{\beta(1-s)^{\alpha-1}}{\Gamma(\alpha)} .
$$

Since $2<\alpha \leq 3$ and

(i) $\alpha-1>1, \beta \in(0,1) \Rightarrow \beta^{\alpha-1}<\beta$,

(ii) $s \leq \beta \Rightarrow s / \beta \leq 1 \Rightarrow 1-(s / \beta) \geq 0$,

(iii) $\beta<1 \Rightarrow 1<1 / \beta \Rightarrow-s(1 / \beta)<-s \Rightarrow 1-s(1 / \beta)<$ $1-s$

thus, we have

$$
\left(1-\frac{s}{\beta}\right)^{\alpha-1}<(1-s)^{\alpha-1} .
$$

From (20), we obtain

$$
\begin{aligned}
(\beta-s)^{\alpha-1} & =\left(\beta\left(1-\frac{s}{\beta}\right)\right)^{\alpha-1} \\
& =\beta^{\alpha-1}\left(1-\frac{s}{\beta}\right)^{\alpha-1} \\
& \leq \beta\left(1-\frac{s}{\beta}\right)^{\alpha-1} \\
& <\beta(1-s)^{\alpha-1} .
\end{aligned}
$$


It follows from (20), (21), and (23), that item 2 in the proof hold.

(b) If $\beta \leq s<1$,

$$
\begin{gathered}
\min _{\beta \leq t \leq 1} G_{1}(t, s)=\frac{\beta(\alpha-1)(1-s)^{\alpha-2}}{\Gamma(\alpha)}, \\
\beta G_{1}(1, s)=\frac{\beta(\alpha-1)(1-s)^{\alpha-2}}{\Gamma(\alpha)}-\frac{\beta(1-s)^{\alpha-1}}{\Gamma(\alpha)} .
\end{gathered}
$$

It follows from (24) that item 2 in the proof holds.

Lemma 8 (see [10]). The unique solution $u(t)$ of (1), (2) is nonnegative and satisfies

$$
\min _{\beta \leq t \leq 1} u(t) \geq \beta\|u\|
$$

Define the cone $K$ by

$$
K=\left\{u \in C[0,1]: u(t) \geq 0, \min _{\beta \leq t \leq 1} u(t) \geq \beta\|u\|\right\}
$$

and the operator $T: K \rightarrow E$ by

$$
\begin{aligned}
T u(t)= & \int_{0}^{1} G_{1}(t, s) \varphi_{q}\left(\int_{0}^{s} a(\tau) f(\tau, u(\tau)) d \tau\right) d s \\
& +\frac{\gamma t}{1-\gamma} \int_{0}^{1} G_{2}(\eta, s) \varphi_{q}\left(\int_{0}^{s} a(\tau) f(\tau, u(\tau)) d \tau\right) d s .
\end{aligned}
$$

Remark 9. By Lemma 6, the problem (1)-(2) has a positive solution $u(t)$ if and only if $u$ is a fixed point of $T$.

Lemma 10. $T$ is completely continuous and $T(K) \subseteq K$.

Proof. By Lemma 8, $T(K) \subseteq K$. In view of the assumption of nonnegativeness and continuity of functions $G_{i}(x, y)$ with $i=1,2$ and $a(t) f(t, u(t))$, we conclude that $T: K \rightarrow K$ is continuous.

Let $\Omega \subseteq K$ be bounded; that is, there exists $M>0$ such that $\|u\| \leq M$ for all $u \in \Omega$.

Let

$$
L=\max _{0 \leq t \leq 1,0 \leq u \leq M}|f(t, u)|
$$

Then from $u \in \Omega$ and from Lemmas 6 and 7, we have

$$
\begin{aligned}
|T u(t)|=\mid \int_{0}^{1} G_{1}(t, s) \varphi_{q}\left(\int_{0}^{s} a(\tau) f(\tau, u) d \tau\right) d s \\
\quad+\frac{\gamma t}{1-\gamma} \int_{0}^{1} G_{2}(\eta, s) \\
\quad \times \varphi_{q}\left(\int_{0}^{s} a(\tau) f(\tau, u) d \tau\right) d s \mid
\end{aligned}
$$

$$
\begin{aligned}
& \leq \int_{0}^{1} G_{1}(1, s) \varphi_{q}\left(\int_{0}^{1} a(\tau) L d \tau\right) d s \\
& +\frac{\gamma}{1-\gamma} \int_{0}^{1} G_{2}(\eta, s) \\
& \times \varphi_{q}\left(\int_{0}^{1} a(\tau) L d \tau\right) d s \\
& \leq L^{q-1} \varphi_{q}\left(\int_{0}^{1} a(\tau) d \tau\right) \\
& \times\left[\int_{0}^{1} \frac{(\alpha-1)}{\Gamma(\alpha)}(1-s)^{\alpha-2} d s+\frac{\gamma}{1-\gamma}\right. \\
& \left.\times \int_{0}^{1} \frac{(\alpha-1)}{\Gamma(\alpha)}(1-s)^{\alpha-2} d s\right] \\
& =\frac{(\alpha-1) L^{q-1}}{(1-\gamma) \Gamma(\alpha)} \varphi_{q}\left(\int_{0}^{1} a(\tau) d \tau\right) \int_{0}^{1}(1-s)^{\alpha-2} d s \\
& =\frac{L^{q-1}}{(1-\gamma) \Gamma(\alpha)} \varphi_{q}\left(\int_{0}^{1} a(\tau) d \tau\right) \doteq l .
\end{aligned}
$$

Hence, $T(\Omega)$ is bounded.

On the other hand, let $u \in \Omega, t_{1}, t_{2} \in[0,1]$ with $t_{1}<t_{2}$; Then

$$
\begin{aligned}
& \left|T u\left(t_{2}\right)-T u\left(t_{1}\right)\right| \\
& \leq L^{q-1}\left[\int_{0}^{1}\left|G_{1}\left(t_{2}, s\right)-G_{1}\left(t_{2}, s\right)\right| \varphi_{q}\right. \\
& \left.\quad \times\left(\int_{0}^{s} a(\tau) f(\tau, u) d \tau\right) d s\right] \\
& +\frac{L^{q-1} \gamma\left|t_{2}-t_{1}\right|}{1-\gamma} \\
& \quad \times \int_{0}^{1} G_{2}(\eta, s) \varphi_{q}\left(\int_{0}^{s} a(\tau) f(\tau, u) d \tau\right) d s .
\end{aligned}
$$

The continuity of $G_{1}$ implies that the right-side of the above inequality tends to zero if $t_{2} \rightarrow t_{1}$. Therefore, $T$ is completely continuous by Arzela-Ascoli theorem.

We introduce the notation

$$
f_{a}:=\liminf _{u \rightarrow a} \min _{\beta \leq t \leq 1} \frac{f(t, u)}{u^{p^{-1}}}, \quad f^{b}:=\operatorname{limsupmax}_{u \rightarrow b} \frac{f(t, u)}{u^{p^{-1}}}
$$


where $a, b=0^{+}, \infty$. Consider the following:

$$
\begin{gathered}
\Lambda_{1}=\left[\beta \left(\int_{\beta}^{1} G_{1}(1, s) \varphi_{q}\left(\int_{0}^{s} a(\tau) d \tau\right) d s+\frac{\gamma}{1-\gamma}\right.\right. \\
\left.\left.\times \int_{\beta}^{1} G_{2}(\eta, s) \varphi_{q}\left(\int_{0}^{s} a(\tau) d \tau\right) d s\right)\right]^{-1} \\
\Lambda_{2}=\left[\frac{1}{(1-\gamma) \Gamma(\alpha)} \varphi_{q}\left(\int_{0}^{1} a(\tau) d \tau\right)\right]^{-1}
\end{gathered}
$$

\section{Single Solutions}

In what follows, the number $\beta \in(0,1)$.

Theorem 11. Suppose that conditions (H1) and (H2) hold. Assume that $f$ also satisfy

$$
\begin{aligned}
& \left(A_{1}\right) f(t, u) \geq(m r)^{p-1}, \beta r \leq u \leq r, \beta \leq t \leq 1, \\
& \left(A_{2}\right) f(t, u) \leq(M R)^{p-1}, 0 \leq u \leq R, 0 \leq t \leq 1,
\end{aligned}
$$

where $m \in\left(\Lambda_{1}, \infty\right)$ and $M \in\left(0, \Lambda_{2}\right)$. Then (1)-(2) has at least one positive solution $u$ such that $r \leq\|u\| \leq R$.

Proof. Without loss of generality, we suppose that $r<R$. For any $u \in K$, we have

$$
u(t) \geq \beta\|u\|, \quad \beta \leq t \leq 1 .
$$

We define two open subsets of $E$,

$$
\Omega_{1}=\{u \in K:\|u\|<r\}, \quad \Omega_{2}=\{u \in K:\|u\|<R\} .
$$

For $u \in \partial \Omega_{1}$, by (33), we have

$$
r=\|u\| \geq u(t) \geq \beta\|u\|=\beta r, \quad t \in[\beta, 1] .
$$

For $t \in[\beta, 1]$, by $\left(A_{1}\right),(27)$, and Lemma 7 , we have

$$
\begin{array}{r}
T u(t)=\int_{0}^{1} G_{1}(t, s) \varphi_{q}\left(\int_{0}^{s} a(\tau) f(\tau, u(\tau)) d \tau\right) d s \\
+\frac{\gamma t}{1-\gamma} \int_{0}^{1} G_{2}(\eta, s) \varphi_{q} \\
\quad \times\left(\int_{0}^{s} a(\tau) f(\tau, u(\tau)) d \tau\right) d s
\end{array}
$$

$$
\begin{aligned}
& \geq \int_{\beta}^{1} \beta G_{1}(1, s) \varphi_{q}\left(\int_{0}^{s} a(\tau)(m r)^{p-1} d \tau\right) d s \\
& \quad+\frac{\gamma \beta}{1-\gamma} \int_{\beta}^{1} G_{2}(\eta, s) \varphi_{q}\left(\int_{0}^{s} a(\tau)(m r)^{p-1} d \tau\right) d s \\
& =r m \beta\left[\int_{\beta}^{1} G_{1}(1, s) \varphi_{q}\left(\int_{0}^{s} a(\tau) d \tau\right) d s\right. \\
& \left.\quad+\frac{\gamma}{1-\gamma} \int_{\beta}^{1} G_{2}(\eta, s) \varphi_{q}\left(\int_{0}^{s} a(\tau) d \tau\right) d s\right] \\
& >r=\|u\| .
\end{aligned}
$$

Therefore,

$$
\|T u\|>\|u\|, \quad \forall u \in \partial \Omega_{1} \cap K .
$$

Then by Theorem 4,

$$
i\left(T, \Omega_{1}, K\right)=0 .
$$

On the other hand, as $u \in \partial \Omega_{2}$, we have

$$
0 \leq u(t) \leq\|u\|=R,
$$

thus, by $\left(A_{2}\right),(27)$, Lemma 7, and (29), we have

$$
\begin{aligned}
T u(t)= & \int_{0}^{1} G_{1}(t, s) \varphi_{q}\left(\int_{0}^{s} a(\tau) f(\tau, u(\tau)) d \tau\right) d s \\
& +\frac{\gamma t}{1-\gamma} \int_{0}^{1} G_{2}(\eta, s) \varphi_{q}\left(\int_{0}^{s} a(\tau) f(\tau, u(\tau)) d \tau\right) d s \\
\leq & M R\left[\int_{0}^{1} G_{1}(t, s) \varphi_{q}\left(\int_{0}^{s} a(\tau) d \tau\right) d s\right. \\
& \left.+\frac{\gamma t}{1-\gamma} \int_{0}^{1} G_{2}(\eta, s) \varphi_{q}\left(\int_{0}^{s} a(\tau) d \tau\right) d s\right] \\
\leq & M R\left[\int_{0}^{1} G_{1}(t, s) \varphi_{q}\left(\int_{0}^{1} a(\tau) d \tau\right) d s\right. \\
& \left.+\frac{\gamma t}{1-\gamma} \int_{0}^{1} G_{2}(\eta, s) \varphi_{q}\left(\int_{0}^{1} a(\tau) d \tau\right) d s\right] \\
\leq & \frac{M R}{(1-\gamma) \Gamma(\alpha)} \varphi_{q}\left(\int_{0}^{1} a(\tau) d \tau\right) d s \\
< & R=\|u\| .
\end{aligned}
$$

Therefore,

$$
\|T u\|<\|u\|, \quad \forall u \in \partial \Omega_{2} \cap K .
$$

Then by Theorem 4,

$$
i\left(T, \Omega_{1}, K\right)=1 .
$$


By (38) and (42):

$$
i\left(T, \Omega_{2} \backslash \overline{\Omega_{1}}, K\right)=1
$$

Then, $T$ has a fixed point $u \in \Omega_{2} \backslash \overline{\Omega_{1}}, u$ is positive solution of problem (1)-(2), and $r<\|u\|<R$.

Corollary 12. Suppose that conditions (H1) and (H2) hold. Assume that $f$ also satisfy

$$
\begin{aligned}
& \left(A_{3}\right) f_{\infty}=\lambda \in\left(\left(2 \Lambda_{1} / \beta\right)^{p-1}, \infty\right), \\
& \left(A_{4}\right) f^{0}=\psi \in\left[0,\left(\Lambda_{2} / 2\right)^{p-1}\right) .
\end{aligned}
$$

Then (1)-(2) has at least one positive solution $u$ such that $r \leq\|u\| \leq R$.

Proof. By $\left(A_{4}\right)$, for $\varepsilon=\left(\Lambda_{2} / 2\right)^{p-1}-\psi$, there exists a suitably small positive number $H_{1}$, as $0<u \leq H_{1}$ and $0 \leq t \leq 1$, such that

$$
\begin{aligned}
f(t, u) \leq(\psi+\varepsilon) u^{p-1} & \leq\left(\frac{\Lambda_{2}}{2}\right)^{p-1} u^{p-1} \\
& \leq\left(\frac{\Lambda_{2}}{2} H_{1}\right)^{p-1} .
\end{aligned}
$$

Let $R=H_{1}$ and $M=\left(\Lambda_{2} / 2\right) \in\left(0, \Lambda_{2}\right)$, then, by (44), condition $\left(A_{2}\right)$ holds.

$\operatorname{By}\left(A_{3}\right)$, for $\varepsilon=\lambda-\left(2 \Lambda_{1} / \beta\right)^{p-1}$, there exists a sufficiently large $r \neq R$ such that

$$
\begin{gathered}
\frac{f(t, u)}{u^{p-1}} \geq \lambda-\varepsilon=\left(\frac{2 \Lambda_{1}}{\beta}\right)^{p-1}, \\
\beta r \leq u<\infty, \quad \beta \leq t \leq 1 .
\end{gathered}
$$

Thus, when $\beta r \leq u \leq r$, one has

$$
f(t, u) \geq\left(\frac{2 \Lambda_{1}}{\beta}\right)^{p-1} u^{p-1} \geq\left(2 \Lambda_{1} r\right)^{p-1} .
$$

Let $m=2 \Lambda_{1} \in\left(\Lambda_{1}, \infty\right)$. Then, by (46), condition $\left(A_{1}\right)$ holds.

Hence, from Theorem 11 the desired result hold.

Corollary 13. Suppose that conditions (H1) and (H2) hold. Assume that $f$ also satisfy

$$
\begin{aligned}
& \left(A_{5}\right) f_{0}=\lambda \in\left(\left(2 \Lambda_{1} / \beta\right)^{p-1}, \infty\right), \\
& \left(A_{6}\right) f^{\infty}=\psi \in\left[0,\left(\Lambda_{2} / 2\right)^{p-1}\right) .
\end{aligned}
$$

Then (1)-(2) has at least one positive solution $u$ such that $r \leq\|u\| \leq R$.

Proof. By $\left(A_{5}\right)$, for $\varepsilon=\lambda-\left(2 \Lambda_{1} / \beta\right)^{p-1}$, there exists a sufficiently small $r>0$, such that

$$
\frac{f(t, u)}{u^{p-1}} \geq \lambda-\varepsilon=\left(\frac{2 \Lambda_{1}}{\beta}\right)^{p-1}, \quad 0<u<r, \beta \leq t \leq 1 .
$$

Thus, when $\beta r \leq u \leq r$, we have

$$
f(t, u) \geq\left(\frac{2 \Lambda_{1}}{\beta}\right)^{p-1} u^{p-1} \geq\left(2 \Lambda_{1} r\right)^{p-1} .
$$

Let $m=2 \Lambda_{1} \in\left(\Lambda_{1}, \infty\right)$. Then by (48), condition $\left(A_{1}\right)$ holds.

By $\left(A_{6}\right)$, for $\varepsilon=\left(\Lambda_{2} / 2\right)^{p-1}-\psi$, there exist a sufficiently large $H_{2} \neq r$ such that

$$
\frac{f(t, u)}{u^{p-1}} \leq \psi+\varepsilon=\left(\frac{\Lambda_{2}}{2}\right)^{p-1}, \quad H_{2} \leq u<\infty, 0 \leq t \leq 1 .
$$

We consider the following two cases.

(a) Suppose that $f(t, u)$ is unbounded, then we know from $(H 1)$ that there is a $R \neq r\left(>H_{2}\right)$ such that

$$
f(t, u) \leq f(t, R), \quad 0 \leq u \leq R, 0 \leq t \leq 1 .
$$

Since $R>H_{2}$, then from (49) and (50) we have

$$
\begin{gathered}
f(t, u) \leq f(t, R) \leq\left(\frac{\Lambda_{2}}{2} R\right)^{p-1}, \\
H_{2} \leq u \leq R, \quad 0 \leq t \leq 1 .
\end{gathered}
$$

Letting $M=\left(\Lambda_{2} / 2\right) \in\left(0, \Lambda_{2}\right)$, we have

$$
f(t, u) \leq(M R)^{p-1}, \quad 0 \leq u \leq R, 0 \leq t \leq 1 .
$$

Thus, $\left(A_{2}\right)$ holds.

(b) Suppose that $f(t, u)$ is bounded, say

$$
f(t, u) \leq L^{p-1}, \quad 0 \leq u<\infty, 0 \leq t \leq 1
$$

In this case, taking sufficiently large $R \geq\left(2 / \Lambda_{2}\right) L$, then letting $M=\Lambda_{2} / 2 \in\left(0, \Lambda_{2}\right)$, we have

$$
\begin{aligned}
f(t, u) \leq L^{p-1} & \leq\left(\frac{\Lambda_{2}}{2} R\right)^{p-1} \leq(M R)^{p-1} \\
0 & \leq u \leq R, \quad 0 \leq t \leq 1 .
\end{aligned}
$$

Thus, $\left(A_{2}\right)$ holds.

Hence, from Theorem 11 the desired result holds.

\section{Multiple Solutions}

To show the existence of multiple solutions we will use the Leggett-Williams fixed point theorem [11]. To this end define the following subsets of a cone $K$ as

$$
\begin{gathered}
K_{c}=\{u \in K:\|u\|<c\}, \\
K(\psi, b, d)=\{u \in K: b \leq \psi(u),\|u\| \leq d\} .
\end{gathered}
$$

Definition 14. A map $\alpha: K \rightarrow[0,+\infty)$ is said to be a nonnegative continuous concave functional on a cone $K$ of a real Banach space $E$ if $\alpha$ is continuous and

$$
\alpha(t x+(1-t) y) \geq t \alpha(x)+(1-t) \alpha(y)
$$

for all $x, y \in K$ and $t \in[0,1]$. 
Theorem 15 (see [11]). Suppose $T: \overline{K_{c}} \rightarrow \overline{K_{c}}$ is completely continuous and suppose that there exists a concave positive functional $\psi$ on $K$ such that $\psi(u) \leq\|u\|$ for $u \in \overline{K_{c}}$. Suppose that there exist constants $0<a<b<d \leq c$ such that

(B1) $\{u \in K(\psi, b, d): \psi(u)>b\} \neq \emptyset$ and $\psi(T u)>b$ if $u \in$ $K(\psi, b, d)$,

(B2) $\|T u\|<a$ if $u \in K_{a}$,

(B3) $\psi(T u)>b$ for $u \in K(\psi, b, c)$ with $\|T u\|>d$.

Then, $T$ has at least three fixed points $u_{1}, u_{2}$, and $u_{3}$ such that $\left\|u_{1}\right\|<a, b<\psi\left(u_{2}\right)$ and $\left\|u_{3}\right\|>a$ with $\psi\left(u_{3}\right)<b$.

Theorem 16. Suppose that there exist $a, b, c$ with $0<a<\beta b<$ $b \leq c$ such that
(C1) $f(t, u)<\left(a \Lambda_{2}\right)^{p-1},(t, u) \in[0,1] \times[0, a]$,
(C2) $f(t, u)>\left(b \Lambda_{1}\right)^{p-1},(t, u) \in[\beta, 1] \times[\beta b, b]$,
(C3) $f(t, u)<\left(c \Lambda_{2}\right)^{p-1},(t, u) \in[0,1] \times[0, c]$.

Then (1)-(2) has at least three positive solutions.

Proof. By Lemma 10, $T: K \rightarrow K$ is completely continuous. Let

$$
\psi(u)=\min _{\beta \leq t \leq 1} u(t) .
$$

It is obvious that $\psi$ is a nonnegative continuous concave functional on $K$ with $\psi(u) \leq\|u\|$, for $u \in \overline{K_{c}}$. Now we will show that the conditions of Theorem 15 are satisfied. For $u \in \overline{K_{c}}$, then $\|u\| \leq c$. For $t \in[0,1]$ by (27), (29), and (C3), one has

$$
\begin{aligned}
T u(t)= & \int_{0}^{1} G_{1}(t, s) \varphi_{q}\left(\int_{0}^{s} a(\tau) f(\tau, u) d \tau\right) d s+\frac{\gamma t}{1-\gamma} \\
& \times \int_{0}^{1} G_{2}(\eta, s) \varphi_{q}\left(\int_{0}^{s} a(\tau) f(\tau, u) d \tau\right) d s \\
< & \int_{0}^{1} G_{1}(t, s) \varphi_{q}\left(\int_{0}^{1} a(\tau)\left(c \Lambda_{2}\right)^{p-1} d \tau\right) d s+\frac{\gamma}{1-\gamma} \\
& \times \int_{0}^{1} G_{2}(\eta, s) \varphi_{q}\left(\int_{0}^{1} a(\tau)\left(c \Lambda_{2}\right)^{p-1} d \tau\right) d s \\
< & \frac{c \Lambda_{2}}{(1-\gamma) \Gamma(\alpha)} \varphi_{q}\left(\int_{0}^{1} a(\tau) d \tau\right)=c .
\end{aligned}
$$

This implies $T: \overline{K_{c}} \rightarrow K_{c}$. By the same method, if $u \in \overline{K_{a}}$, then we can get $\|T u\|<a$ and therefore (B2) is satisfied. Next, we assert that $\{u \in K(\psi, \beta b, b): \psi(u)>\beta b\} \neq \phi$ and $\psi(T u)>$ $\beta b$ for all $u \in K(\psi, \beta b, b)$. In fact, the constant function

$$
\frac{\beta b+b}{2} \in\{u \in K(\psi, \beta b, b): \psi(u)>\beta b\} .
$$

On the other hand, for $u \in K(\psi, \beta b, b)$, we have

$$
\beta b \leq \psi(u)=\min _{\beta \leq t \leq 1} u(t) \leq u(t) \leq\|u\| \leq b, \quad \forall t \in[\beta, 1] .
$$

Thus, in view of (27), Lemma 7, and (C2), one has

$\psi(T u)$

$$
\begin{gathered}
=\min _{\beta \leq t \leq 1}\left[\int_{0}^{1} G_{1}(t, s) \varphi_{q}\left(\int_{0}^{s} a(\tau) f(\tau, u(\tau)) d \tau\right) d s+\frac{\gamma t}{1-\gamma}\right. \\
\left.\times \int_{0}^{1} G_{2}(\eta, s) \varphi_{q}\left(\int_{0}^{s} a(\tau) f(\tau, u(\tau)) d \tau\right) d s\right] \\
\geq \int_{\beta}^{1} \beta G_{1}(1, s) \varphi_{q}\left(\int_{0}^{s} a(\tau) f(\tau, u(\tau)) d \tau\right) d s \\
+\frac{\beta \gamma}{1-\gamma} \int_{\beta}^{1} G_{2}(\eta, s) \varphi_{q}\left(\int_{0}^{s} a(\tau) f(\tau, u(\tau)) d \tau\right) d s \\
>b \Lambda_{1} \beta\left[\int_{\beta}^{1} G_{1}(1, s) \varphi_{q}\left(\int_{0}^{s} a(\tau) d \tau\right) d s\right. \\
\left.+\frac{\gamma}{1-\gamma} \int_{\beta}^{1} G_{2}(\eta, s) \varphi_{q}\left(\int_{0}^{s} a(\tau) d \tau\right) d s\right]=b
\end{gathered}
$$

$>\beta b$

as required and therefore $(B 1)$ is satisfied.

Finally, we assert that if $u \in K(\psi, \beta b, c)$ with $\|T u\|>b$ then $\psi(u)>\beta b$. To see this, suppose that $u \in K(\psi, \beta b, c)$ and $\|T u\|>b$, then it follows from Lemma 10 that

$$
\psi(T u)=\min _{\beta \leq t \leq 1}(T u)(t) \geq \beta\|T u\|>\beta b .
$$

Thus, (B3) is satisfied.

Therefore, by the conclusion of Theorem 15, the operator $T$ has at least three fixed points. This implies that (1)-(2) has at least three solutions.

\section{Examples}

Example 1. Consider the boundary value problem with $p$ Laplacian:

$$
\begin{gathered}
\left(\varphi_{p}\left(D_{0^{+}}^{5 / 2} u(t)\right)\right)^{\prime}+\frac{1}{4} t^{-1 / 2} \frac{a u^{1 / 2} e^{2 u}}{b+e^{u}+e^{2 u}}=0, \\
0<t<1, \\
D_{0^{+}}^{5 / 2} u(0)=u(0)=u^{\prime \prime}(0)=0, \quad u^{\prime}(1)=\frac{8}{9} u^{\prime}\left(\frac{1}{2}\right),
\end{gathered}
$$

where $\alpha=5 / 2, \eta=1 / 2, \gamma=8 / 9, \beta=1 / 2, p=3 / 2, q=$ 3, $f(t, u)=a u^{1 / 2} e^{2 u} /\left(b+e^{u}+e^{2 u}\right), a(t)=(1 / 4) t^{-1 / 2}$, and $\varphi_{3}\left(\int_{0}^{1} a(t) d t\right)=1 / 4, \varphi_{3}\left(\int_{0}^{s} a(t) d t\right)=(1 / 4) s$.

Then

$$
f^{0}=\psi=\frac{a}{b+2}, \quad f_{\infty}=\lambda=a .
$$


Next,

$$
\begin{aligned}
\Lambda_{1} & =\left[\frac{1}{2}\left(\int_{1 / 2}^{1} G_{1}(1, s) \frac{1}{4} s d s+8 \int_{1 / 2}^{1} G_{2}\left(\frac{1}{2}, s\right) \frac{1}{4} s d s\right)\right]^{-1} \\
& =\left[\frac{9}{35} \sqrt{\frac{2}{\pi}}\right]^{-1} \approx 4.873999426 .
\end{aligned}
$$

Then, for $a=5$ we have $f_{\infty} \in\left(\left(2 \Lambda_{1} / \beta\right)^{p-1}, \infty\right)$, so condition $\left(A_{3}\right)$ holds.

Now, Consider

$$
\Lambda_{2}=\frac{\sqrt{\pi}}{3} \approx 0.5908179503 .
$$

Then, for $a=5$ and $b=8$ we have $f^{0} \in\left[0,\left(\Lambda_{2} / 2\right)^{p-1}\right)$, so condition $\left(A_{4}\right)$ holds. Therefore, by Corollary $12,(63)$ has at least one positive solution.

Example 2. Consider the boundary value problem with $p$ Laplacian:

$$
\begin{gathered}
\left(\varphi_{p}\left(D_{0^{+}}^{5 / 2} u(t)\right)\right)^{\prime}+\frac{1}{4} t^{-1 / 2} f(t, u)=0, \quad 0<t<1, \\
D_{0^{+}}^{5 / 2} u(0)=u(0)=u^{\prime \prime}(0)=0, \quad u^{\prime}(1)=\frac{8}{9} u^{\prime}\left(\frac{1}{2}\right),
\end{gathered}
$$

where $\alpha=5 / 2, \eta=1 / 2, \gamma=8 / 9, \beta=1 / 2, p=3 / 2, q=3$, $a(t)=(1 / 4) t^{-1 / 2}, \varphi_{3}\left(\int_{0}^{1} a(t) d t\right)=1 / 4$, and $\varphi_{3}\left(\int_{0}^{s} a(t) d t\right)=$ $(1 / 4) s$.

Let

$$
f(t, u)= \begin{cases}\frac{t}{40}+14 u^{2}, & u \leq 1 \\ 13+\frac{t}{40}+u^{1 / 4}, & u>1\end{cases}
$$

By Example 1 , we have $\Lambda_{1} \approx 4.873999426$ and $\Lambda_{2} \approx$ 0.5908179503 .

Choosing $a=1 / 14, b=18$, and $c=1296$, then

$$
\begin{gathered}
f(t, u)=\frac{t}{40}+14 u^{2} \approx 0.096 \ldots<\left(a \Lambda_{2}\right)^{1 / 2} \approx 0.20 \ldots, \\
(t, u) \in[0,1] \times\left[0, \frac{1}{14}\right], \\
f(t, u)=\frac{t}{40}+13+u^{1 / 4} \approx 14.7 \ldots>\left(9 \Lambda_{1}\right)^{1 / 2} \approx 9.3 \ldots, \\
(t, u) \in\left[\frac{1}{2}, 1\right] \times[9,18], \\
f(t, u)=\frac{t}{40}+13+u^{1 / 4} \approx 19 \ldots<\left(1296 \Lambda_{2}\right)^{1 / 2} \approx 27 \ldots
\end{gathered}
$$$$
(t, u) \in[0,1] \times[0,1296] \text {. }
$$

Then the conditions $(\mathrm{C} 1)-(\mathrm{C} 3)$ are satisfied. Therefore, it follows from Theorem 16 that (67) has at least three positive solutions $u_{1}, u_{2}$, and $u_{3}$ such that

$$
\begin{aligned}
& \left\|u_{1}\right\|<\frac{1}{14}, \quad 18<\psi\left(u_{2}\right), \quad\left\|u_{3}\right\|>\frac{1}{14} \\
& \text { with } \psi\left(u_{3}\right)<18 .
\end{aligned}
$$

\section{Acknowledgment}

Francisco J. Torres was partially supported by DIUDA 221231, Universidad de Atacama.

\section{References}

[1] R. Liang, J. Peng, and J. Shen, "Double positive solutions for a nonlinear four-point boundary value problem with a $p$-Laplacian operator," Nonlinear Analysis: Theory, Methods \& Applications, vol. 323, pp. 413-425, 2006.

[2] D. Zhao, H. Wang, and W. Ge, "Existence of triple positive solutions to a class of $p$-Laplacian boundary value problems," Journal of Mathematical Analysis and Applications, vol. 328, no. 2, pp. 972-983, 2007.

[3] G. Chai, "Positive solutions for boundary value problem of fractional differential equation with p-Laplacian operator," Boundary Value Problems, vol. 2012, article 18, 2012.

[4] H. Su, Z. Wei, and B. Wang, "The existence of positive solutions for a nonlinear four-point singular boundary value problem with a $p$-Laplacian operator," Nonlinear Analysis: Theory, Methods \& Applications A, vol. 66, no. 10, pp. 2204-2217, 2007.

[5] H. Su, "Positive solutions for $n$-order $m$-point $p$-Laplacian operator singular boundary value problems," Applied Mathematics and Computation, vol. 199, no. 1, pp. 122-132, 2008.

[6] X. Tang, C. Yan, and Q. Liu, "Existence of solutions of two-point boundary value problems for fractional $p$-Laplace differential equations at resonance," Journal of Applied Mathematics and Computing, vol. 41, no. 1-2, pp. 119-131, 2013.

[7] K. Deimling, Nonlinear Functional Analysis, Springer, Berlin, Germany, 1985.

[8] D. J. Guo and V. Lakshmikantham, Nonlinear Problems in Abstract Cones, Academic Press, San Diego, Calif, USA, 1988.

[9] G. Chai, "Existence results for boundary value problems of nonlinear fractional differential equations," Computers \& Mathematics with Applications, vol. 62, no. 5, pp. 2374-2382, 2011.

[10] F. J. Torres, "Existence of positive solutions for a boundary value problem of a nonlinear fractional differential equation," Bulletin of the Iranian Mathematical Society, vol. 39, no. 2, pp. 307-323, 2013.

[11] R. W. Leggett and L. R. Williams, "Multiple positive fixed points of nonlinear operators on ordered Banach spaces," Indiana University Mathematics Journal, vol. 28, no. 4, pp. 673-688, 1979. 


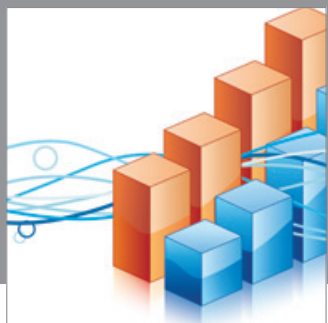

Advances in

Operations Research

mansans

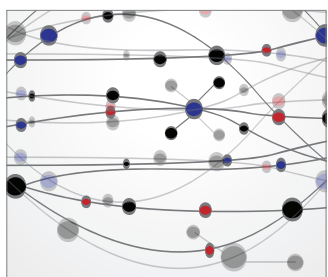

The Scientific World Journal
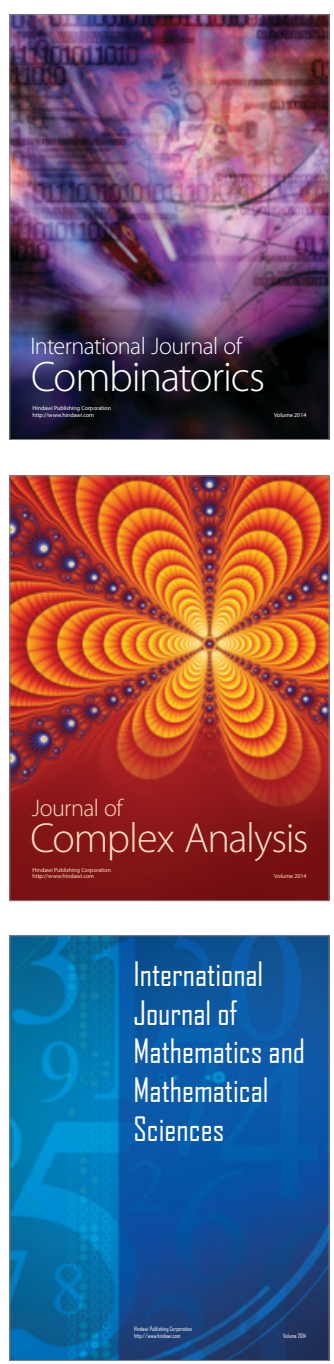
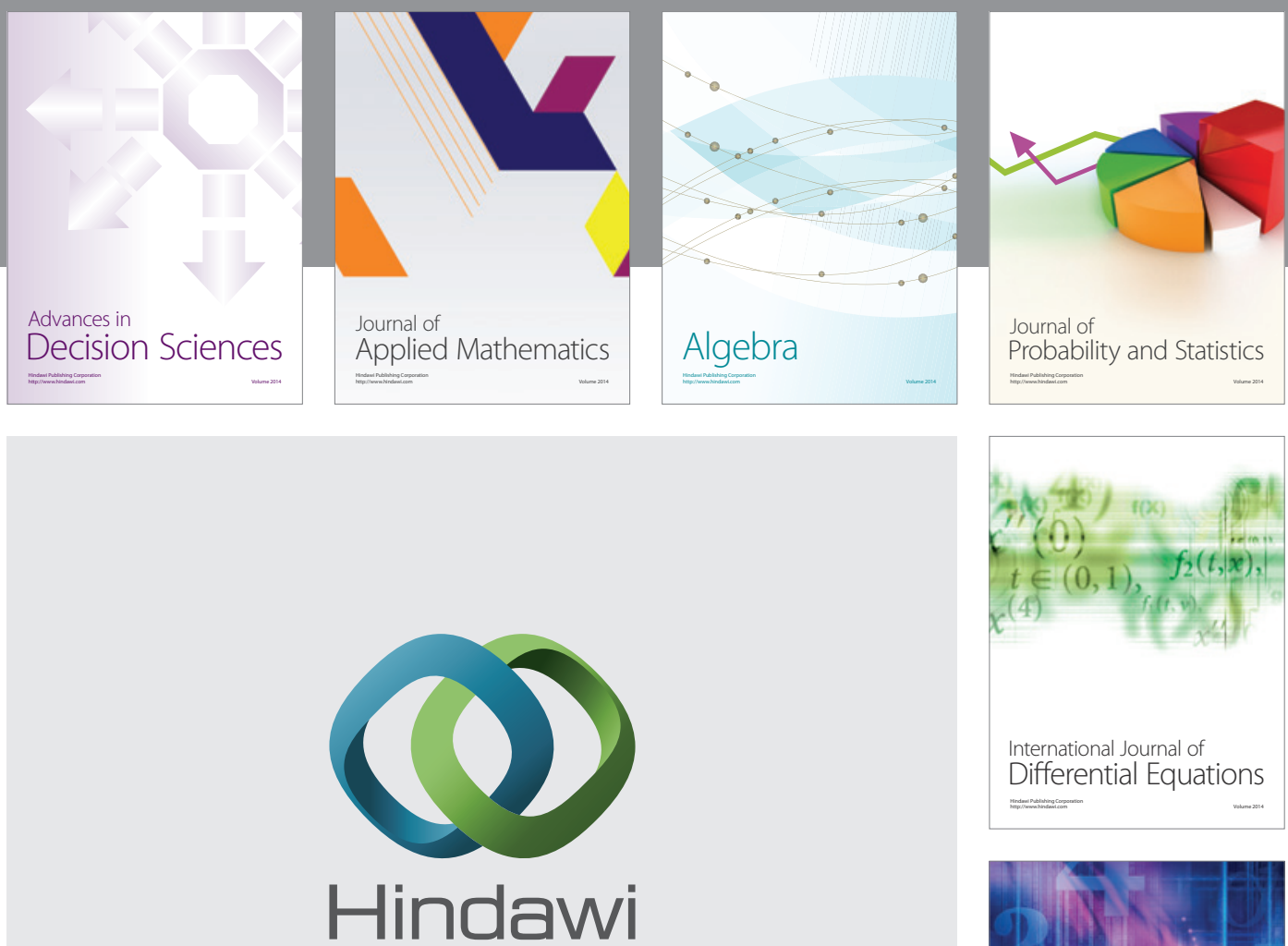

Submit your manuscripts at http://www.hindawi.com
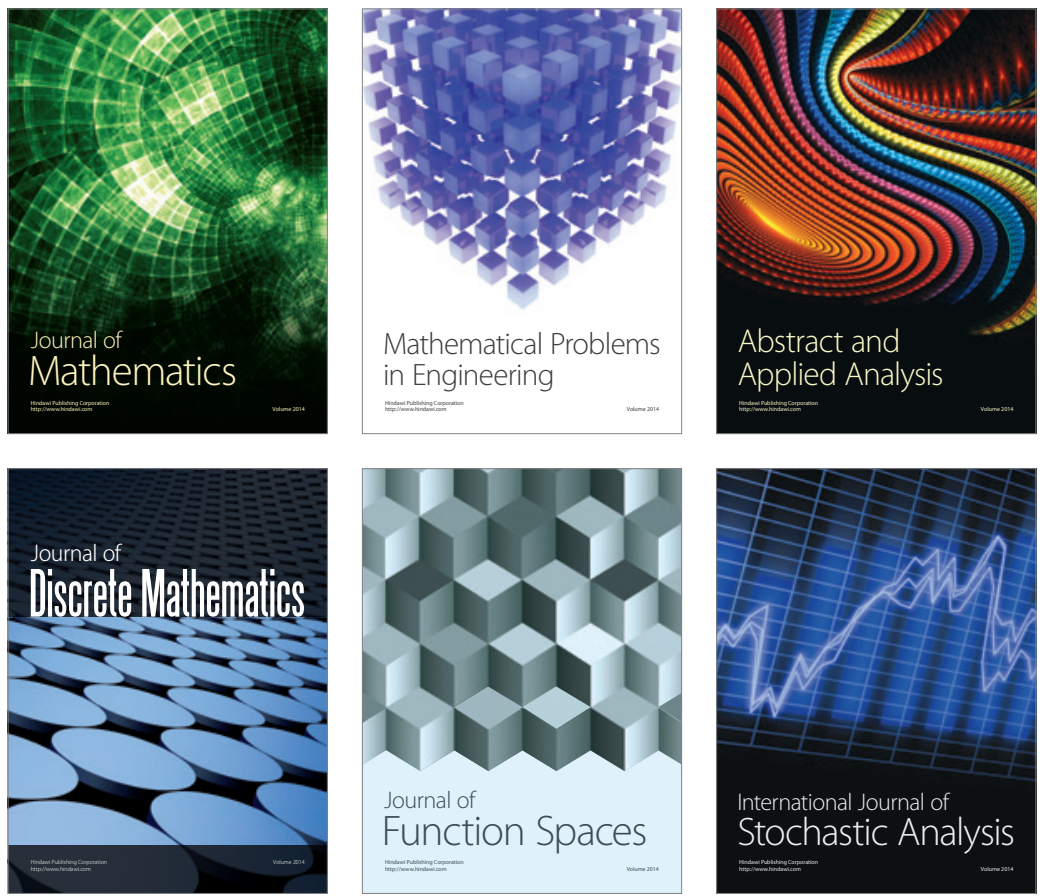

Journal of

Function Spaces

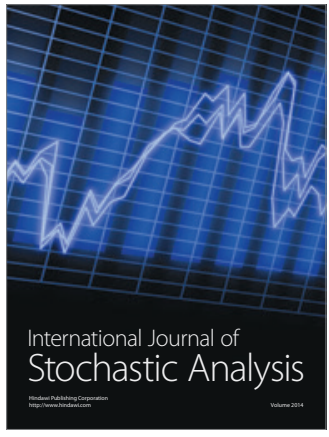

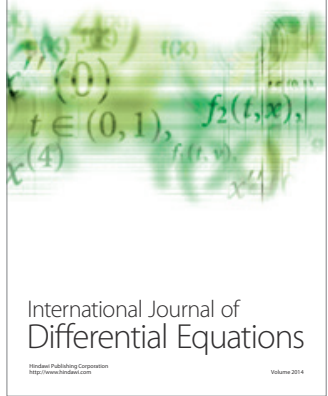
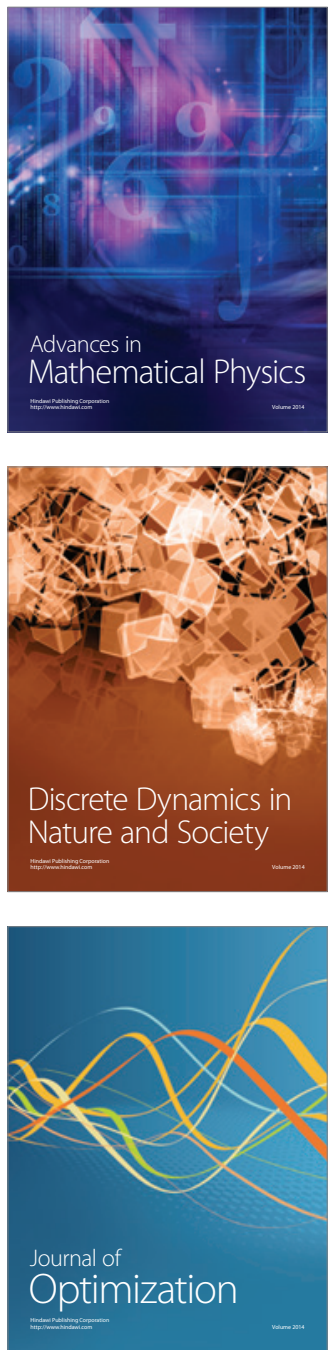